Les déformations rachidiennes sont une des principales complications orthopédiques secondaires à l'hypotonie axiale résultant de nombreuses maladies neuromusculaires. Au premier rang de ces déformations, la scoliose associe en particulier une rotation vertébrale dans le plan horizontal et une convexité dans le plan frontal. On l'observe notamment dans l'amyotrophie spinale infantile. Ces déformations du rachis entraînent une déviation de l'axe du rachis et une obliquité du bassin, les deux phénomènes se surajoutant à l'effondrement global du tronc liée à l'hypotonie musculaire résultant de ces pathologies. II en résulte une majoration de l'insuffisance respiratoire restrictive et une dégradation des capacités fonctionnelles et de la qualité de vie [1]. Le traitement des scolioses liées aux maladies neuromusculaires est complexe, car ces déformations sont souvent précoces, d'évolution rapide, chez des sujets à l'état général altéré. La prise en charge est multidisciplinaire et associe médecins, appareilleurs, chirurgiens orthopédistes et rééducateurs. La chirurgie est le plus communément proposée lorsque le traitement conservateur (kinésithérapie et corsets) est en échec ou en deçà des espérances. II n'existe toutefois aucune étude contrôlée et randomisée comparant la prise en charge orthopédique et la chirurgie des scolioses neuromusculaires, notamment concernant le type de chirurgie et le moment le plus opportun pour la proposer.

Lorsque la scoliose a une évolutivité permettant d'atteindre l'âge de la maturité osseuse, et si l'état général du sujet le permet, le traitement « gold standard » demeure l'arthrodèse vertébrale postérieure [2]. Mais lorsque la déformation est rapidement évolutive, notamment chez de plus jeunes enfants, la fusion vertébrale a pour conséquence l'arrêt de la croissance du tronc et de la cage thoracique, et peut être à l'origine d'autres complications. En forte progression depuis ces deux dernières décennies, de nouvelles techniques opératoires ont été développées et mises sur le marché. Appelées par certaines équipes «tiges de croissance » (« growing rods » ou «fusionless techniques » en anglais), ces systèmes d'instrumentation rachidienne sans fusion sont des techniques moins invasives que l'arthrodèse classique, avec moins de risque de complication per opératoire, et des suites opératoires plus simples. Selon la pathologie et les fonctions motrices résiduelles du patient, elles peuvent être proposées chez des enfants jeunes qui

\section{Les instrumentations rachidiennes sans fusion vertébrale dans les scolioses neuromusculaires}

Étienne Saudeau

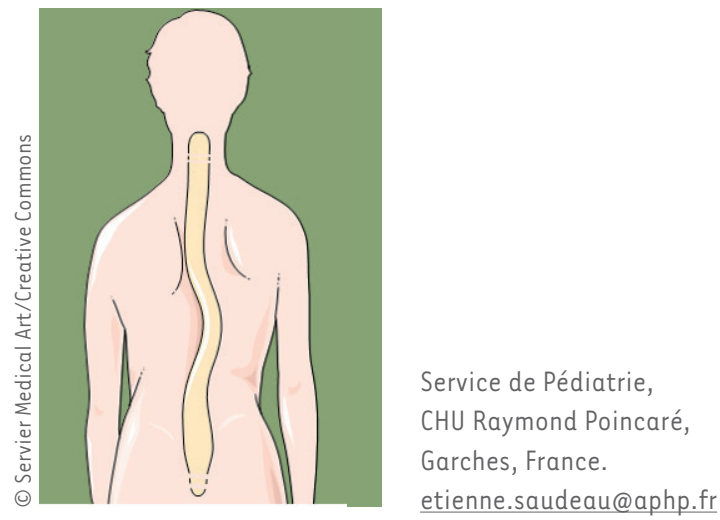

n'ont pas encore atteint leur maturité osseuse, et chez des sujets à l'état général altéré. En corrigeant de façon progressive la déformation du rachis, ces instrumentations « growth friendly » retardent la fusion définitive des vertèbres entre elles et maintiennent, dans une certaine mesure, la croissance du tronc et du thorax, gage d'un développement pulmonaire optimal de l'enfant. Cette correction progressive des déformations du rachis permet aussi de limiter le risque d'atteinte médullaire inhérent aux interventions chirurgicales rachidiennes.

En France, deux techniques sont particulièrement développées : l'une permettant une extension mécanique de tiges en titane, l'autre, leur extension par un moteur à commande magnétique. D'autres techniques existent, d'utilisation plus confidentielle en France pour le traitement des scolioses neuromusculaires, comme l'instrumentation par prothèses costales verticales extensibles en titane («Vertical Expandable Prosthetic Titanium Rib » ou VEPTR), dont les fixations en crochet peuvent se faire entre des côtes, des vertèbres ou le bassin. Nous développerons ici les deux seules techniques de tiges de croissance utilisées en France.

\section{Tiges d'instrumentation mini-invasive sans greffe}

Cette technique de montage « en tour Eiffel » a la particularité de mettre en place, de façon «mini-invasive » et sans arthrodèse, deux tiges coulissantes de part et d'autre du rachis, s'étendant de la vertèbre $\mathrm{Tl}$ jusqu'au bassin, ancrées en proximal par quatre crochets, et en distal par deux vis ilio-sacrées (Figures $I A$ et $1 B$ ). Le temps 


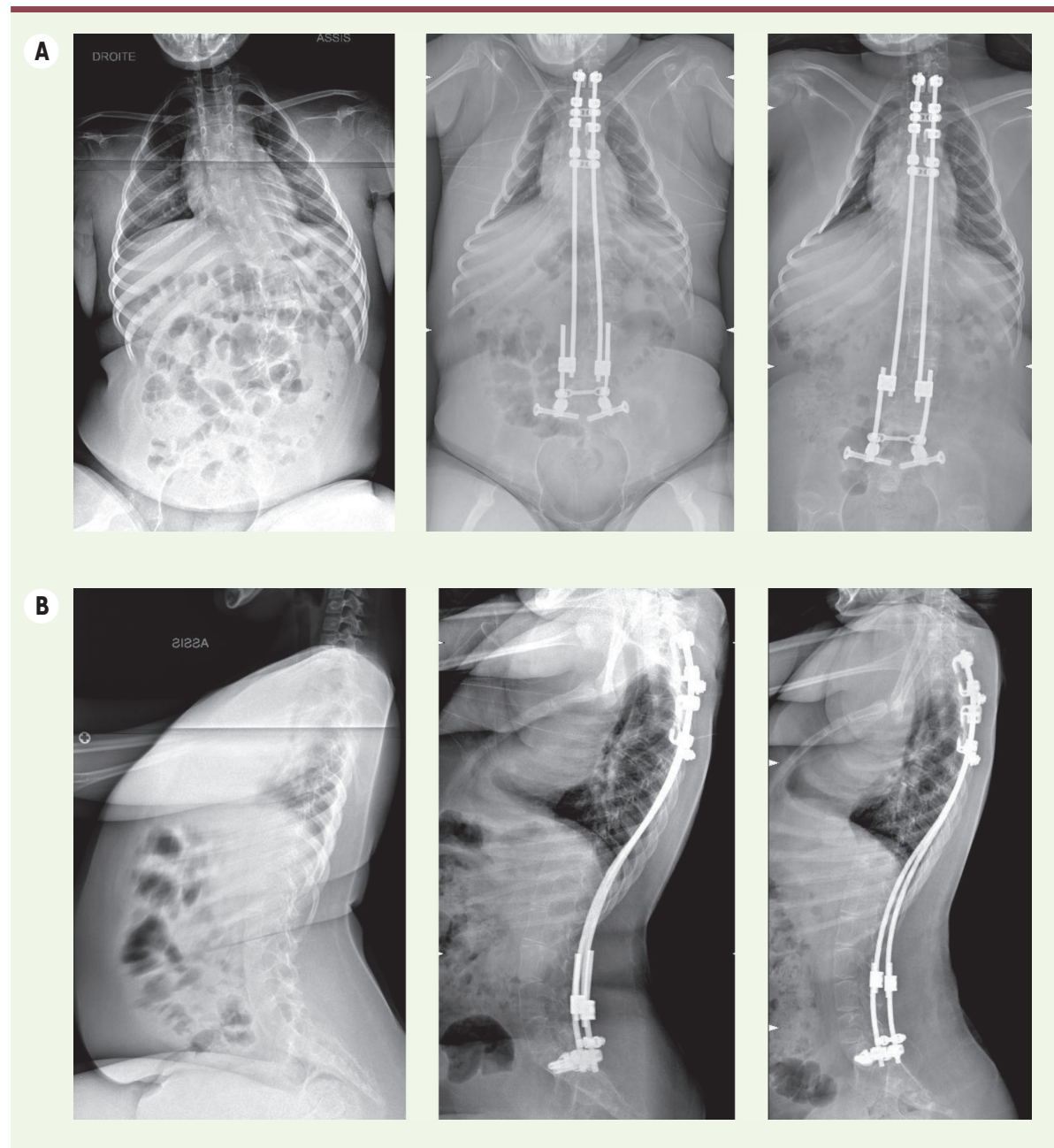

Figure 1. Tiges d'instrumentation mini-invasive sans greffe, pré, post-opératoire immédiat et postallongement. A. Vue de face. B. Vue de profil.

opératoire est plus court que celui d'une arthrodèse vertébrale postérieure classique, et les suites opératoires sont aussi plus simples. Dans certains cas, une à trois nouvelles interventions peuvent être nécessaires pour poursuivre la correction de la déformation rachidienne ou de l'obliquité pelvienne, selon la progression de cette déformation, le degré de bascule en position assise, ou selon la croissance rachidienne de l'enfant. Ces interventions secondaires, plus courtes, font glisser les tiges l'une sur l'autre, de façon symétrique ou non. Jusqu'à présent, il n'a jamais été nécessaire de réaliser une fusion définitive par une arthrodèse rachidienne en fin de croissance, celle-ci se faisant de manière spontanée, les vertèbres ayant tendance à s'enraidir après une dizaine d'années au contact du matériel métallique.

En 2018, Miladi et al. décrivent les résultats d'une série de cent enfants souffrant d'une scoliose neurologique secondaire et opérés selon cette technique [3]. L'âge moyen au moment de la chirurgie initiale était de 11 ans. Ces enfants étaient atteints d'une infirmité motrice cérébrale pour 61 d'entre eux, 22 avaient une amyotrophie spinale, 10 avaient une dystrophie musculaire, et 7 avaient d'autres pathologies neurologiques. Avec un recul moyen de trois ans, l'équipe a objectivé une amélioration de l'angle de Cobb, celui-ci passant de $89^{\circ}$ à $35^{\circ}$ en moyenne, et de l'angle d'obliquité pelvienne passant de $29^{\circ}$ à $5^{\circ}$ en moyenne. Vingt-six patients ont connu des complications, dont douze complications mécaniques et seize infections du site opératoire. Aucune arthrodèse n'a été requise en fin de suivi.

En 2021, la même équipe rapporte les résultats du suivi de 59 enfants atteints d'amyotrophie spinale infantile, tous opérés selon cette technique chirurgicale [4]. D'un âge moyen de 11 ans, ils ont été suivis en moyenne pendant cinq ans. Parmi tous ces enfants, 26 bénéficiaient d'un traitement par nusinersen intrathécal. En moyenne, l'angle de Cobb est passé de 79 à $41^{\circ}$, et celui de l'obliquité pelvienne de 24 à $5^{\circ}$. Bien que l'espace moyen intra-thoracique disponible pour le poumon soit passé de 77 à $93 \%$, il n'y a pas eu de variation significative des chiffres de capacité vitale au cours du suivi. Essentiellement pour des raisons de mauvaise tenue de tête, treize patients ont continué à porter leur corset en post-opératoire. Quatrevingt onze pour cent des patients ont témoigné d'une satisfaction vis-à-vis de l'opération. Sur les neuf patients qui ont subi des complications infectieuses ou mécaniques, seulement un patient a nécessité l'ablation du matériel. Seuls 30 enfants ont nécessité des procédures d'allongement de la tige, avec un intervalle entre les procédures de 1,9 ans en moyenne. Ici encore, aucune arthrodèse définitive n'a été nécessaire chez aucun patient, bien que le recul soit moindre.

Depuis 2020 en France, un montage similaire est utilisé avec des tiges à l'agrandissement mécanique automatique («One-way self-expanding rod »), grâce à un système de jonction des tiges « en crémaillère ». Ainsi, les tiges peuvent accroitre leur longueur et leur potentiel de correction progressive de façon automatique avec la croissance de l'enfant, sans que de nouvelles interventions chirurgicales soient nécessaires [5]. Cette nouvelle technique semble prometteuse, notamment pour les scolioses infantiles, mais de nouvelles études cliniques sont nécessaires pour juger de leur efficacité. 
Tiges de croissances avec contrôle magnétique

L'autre technique chirurgicale sans fusion vertébrale est l'instrumentation par tiges de croissance contrôlées par système motorisé magnétique (MCGRS pour « magnetically controlled growing rods surgery »). Cette technique a l'avantage de ne nécessiter qu'une seule intervention initiale, moteur implanté dans les tiges permettant à celles-ci de s'agrandir par télécommande plusieurs fois par an, de façon synchrone ou non selon l'indication. L'un des systèmes d'implant contrôlé magnétiquement est le MAGEC (MAGnetic Expansion Control ; Ellipse Technologies), commercialisé aux États-Unis depuis 2012 (Figures 2A et 2B).

Dans une étude publiée en 2014, Yoon et al. ont montré une évolution statistiquement favorable des capacités vitales forcées postopératoires (avec un gain moyen de $17 \%$ ) chez six enfants atteints de scolioses neuromusculaires, d'un âge moyen de 7,5 ans et suivis pendant deux ans après une instrumentation MCGRS [6]. Parmi eux, deux enfants avaient une SMA de type II et deux autres avaient une neurofibromatose. Les paramètres orthopédiques ont aussi été significativement améliorés, avec un de Cobb moyen passé de $87^{\circ}$ à $53^{\circ}$ dans le plan coronal, et de $66^{\circ}$ à $30^{\circ}$ dans le plan sagittal.

A
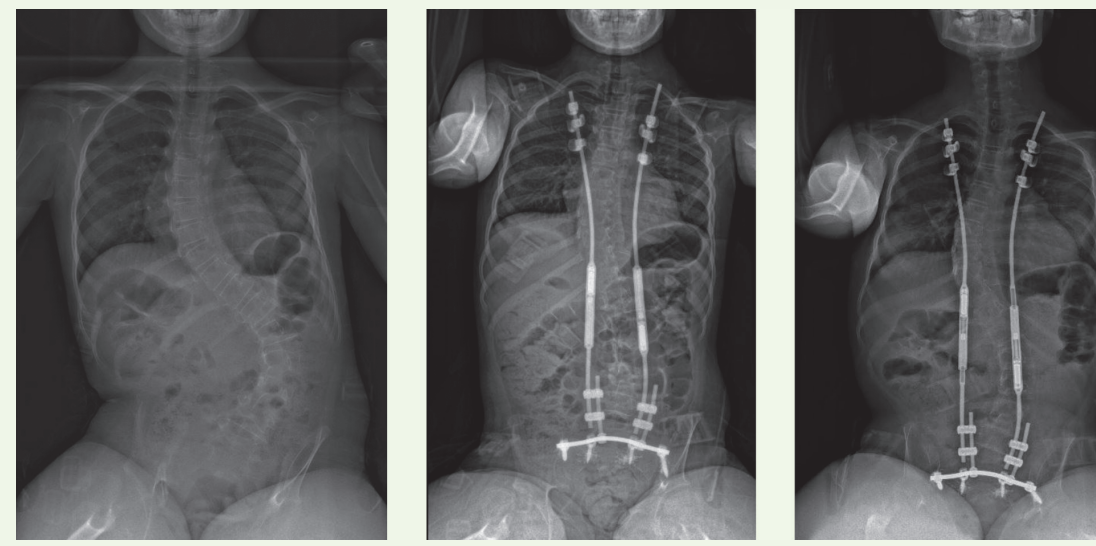

B
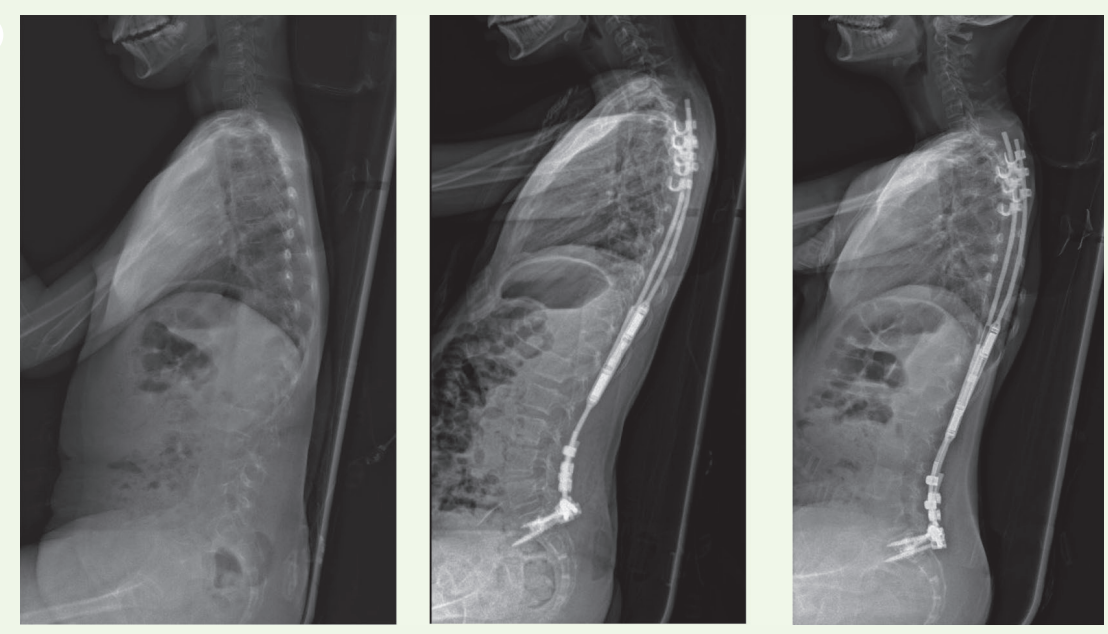

Figure 2A. Tiges de croissances avec contrôle magnétique : pré, post-opératoire immédiat et postallongement. A. Vue de face. B. Vue de profil.
En 2017, Lorenz et al. ont suivi pendant deux ans 21 enfants avec amyotrophie spinale de type II, opérés dès l'âge de 7,8 ans en moyenne avec une instrumentation MCGRS [7]. L'angle de Cobb a été corrigé de $70^{\circ}$ à $30^{\circ}$ et celui de l'obliquité pelvienne de $17^{\circ}$ à $4^{\circ}$, avec maintien de ces corrections sur toute la durée du suivi. Les auteurs notaient également un gain de taille de tronc de $50 \mathrm{~mm}$ lors de la mise en place des tiges, avant un gain de $13,5 \mathrm{~mm}$ par an au cours du traitement avec quatre procédures d'allongement par an. Malheureusement, les auteurs notaient une augmentation progressive de la cyphose thoracique, mais aussi quatre complications générales dont deux ont nécessité un traitement chirurgical, et six échecs de procédures d'allongement dont trois nécessitant une reprise chirurgicale.

En 2020, Swarup et al. ont suivi, sur 15 centres aux USA pendant deux ans, 66 enfants avec amyotrophie spinale infantile, et traités par des prothèses costales verticales extensibles en titane (VEPTR) et des tiges de croissance à commande magnétique (MCGR) [8]. Malgré des corrections de déformation satisfaisantes chez ces enfants âgés en moyenne de 7 ans, le taux de complication est resté relativement élevé : $24 \%$ chez les patients traités par les tiges MCGR, et $45 \%$ pour ceux traités avec le système VEPTR.

Enfin, notons que le système MAGEC (MAGnetic Expansion Control, NuVasive Specialised Orthopaedics, San Diego, USA) était l'objet, encore récemment, de questionnements en matière d'innocuité. Un premier avis de sécurité («Field Safety Notice ») a été publié en juin 2019 en raison de la rupture de la goupille de verrouillage pour $5 \%$ des premiers modèles système MAGEC commercialisés avant mars 2015 [9]. En février 2020, le Royaume-Uni a publié une alerte de matériel médical («Medical Device Alert») en raison du risque de décollement de l'embout des tiges MAGEC Modèle $X$ après leur implantation, avant que ce pays ne décide finalement de suspendre la mise sur le marché de ce seul modèle à partir d'avril 2020 [10]. Une étude 
publiée en février 2021 par une équipe anglaise affirme, sur base d'une étude comprenant 66 patients, la présence de métallose, c'est-à-dire l'accumulation de particules de métal dans les tissus, chez $79 \%$ de ces enfants [11]. Les conséquences de cette métallose ne sont pas connues. En juillet 2021, les USA ont de nouveau autorisé la commercialisation des tiges MAGEC Modèle $X$ après une modification de ces tiges par la société qui les fabrique, et une mise à jour des informations données aux patients [12].

\section{Conclusion}

Ces nouvelles instrumentations rachidiennes ont fait la preuve de leurs nombreux bénéfices en termes d'adaptation du matériel à la croissance du tronc et du thorax, de sécurité d'emploi et de simplicité des suites opératoires, d'efficacité pour la correction des scolioses précoces évolutives avec ou sans obliquité pelvienne, mais aussi de coût économique, chez de nombreux enfants atteints de maladie neuromusculaires, notamment d'amyotrophie spinale infantile.

Cette évolution des techniques opératoires est la bienvenue, en ces temps de révolution thérapeutique, avec l'avènement des nouvelles thérapies par saut d'exon et de la thérapie génique. L'absence de fusion vertébrale permet un accès plus facile aux injections intrathécales de certains de ces traitements, et l'adaptation de l'instrumentation chirurgicale à la croissance est bénéfique pour des enfants traités de plus en plus jeunes par ces nouvelles thérapies pharmacologiques, dont les conséquences en termes de croissance et de déformations rachidiennes sont encore mal connues.

Cependant, il demeure fondamental de poursuivre un suivi multidisciplinaire des patients neuromusculaires, afin de prévenir toutes déformations précoces de certaines articulations (notamment de prévenir les déformations vertébrales cervicales au-dessus du matériel chirurgical) par des assouplissements musculaires et des traitements orthopédiques bien conduits, et par un renforcement musculaire doux des muscles hypotoniques. Ces traitements chirurgicaux étant proposés pour des enfants de plus en plus jeunes, il conviendra de poursuivre notre vigilance clinique tout au long de leur croissance. $\diamond$

\section{SUMMARY}

Fusionless spine instrumentations in neuromuscular scoliosis Less invasive techniques are now available to treat neuromuscular scoliosis efficiently. Rods can be implanted safely and at an early stage to correct and prevent further spine deformities. These techniques are particularly adapted to children with spinal muscular atrophy. The expansion of rods is possible magnetically or mechanically and enables to follow the spine growth timeline optimally. Of note, a risk a metallosis has been reported for some magnetic rods available on the market. $\diamond$

\section{LIENS D'INTÉRÊT}

L'auteur déclare n'avoir aucun lien d'intérêt concernant les données publiées dans cet article.

\section{RÉFÉRENCES}

1. Vialle R, Thévenin-Lemoine C, Mary P. Neuromuscular scoliosis. Orthop Traumatol Surg Res 2013; 99 (suppl 1) : S124-39.

2. Mary $P$, Servais $L$, Vialle R. Neuromuscular diseases: diagnosis and management. Orthop Traumatol Surg Res 2018 ; 104 (suppl 1) : S89-95.

3. Miladi L, Gaume M, Khouri N, et al. Minimally invasive surgery for neuromuscular scoliosis: results and complications in a series of one hundred patients. Spine $2018 ; 43$ : ع968-75.

4. Gaume M, Saudeau $\varepsilon$, Gomez-Garcia de la Banda M, et al. Minimally invasive fusionless surgery for scoliosis in spinal muscular atrophy: long-term follow-up results in a series of 59 patients. J Pediatr Orthop 2021 ; 41 : 549-58

5. Miladi L, Khouri N, Pradon J, et al. One-way self-expanding rod for earlyonset scoliosis: early results of a clinical trial of 20 patients. Eur Spine J $2021 ; 30: 749-58$.

6. Yoon WW, Sedra F, Shah S, et al. Improvement of pulmonary function in children with early-onset scoliosis using magnetic growth rods. Spine 2014 ; 39 : 1196-202.

7. Lorenz HM, Badwan B, Hecker MM, et al. Magnetically controlled devices parallel to the spine in children with spinal muscular atrophy. JB JS Open Access $2017 ; 2$ : e0036.

8. Swarup I, MacAlpine EM, Mayer OH, et al. Impact of growth friendly interventions on spine and pulmonary outcomes of patients with spinal muscular atrophy. Eur Spine J $2021 ; 30: 768-74$.

9. https://www.nuvasive.com/wp-content/uploads/2019/06/Final_FSN_ DATED-25JUN19.pdf

10. https://www.gov.uk/drug-device-alerts/spinal-implant-all-magecsystems-supply-suspended-to-the-uk-mda-2020-011

11. Rushton PRP, Smith SL, Fender D, et al. Metallosis is commonly associated with magnetically controlled growing rods: results from an independent multicentre explant database. Eur Spine J 2021 ; 30 : 1905-11.

12. https://www.nuvasive.com/wp-content/uploads/2021/07/NuVasivestatement_US-availability-of-MAGEC-device_15July2021_Final.pdf 